\title{
Compiler-Guided Identification of Critical Sections in Parallel Code
}

\author{
Stefan Kempf, Ronald Veldema, and Michael Philippsen \\ University of Erlangen-Nuremberg, Computer Science Dept., Programming Systems Group, \\ Martensstr. 3, 91058 Erlangen, Germany \\ \{stefan.kempf, veldema, philippsen\}@cs.fau.de
}

\begin{abstract}
There is a huge body of sequential legacy code that needs to be refactored for multicore processors. Especially for control code for embedded systems it is often easy to split the program into multiple threads. But it is difficult to identify critical sections to avoid data races as the legacy code hides its synchronization in a static schedule, priorities and interrupts.

To ease refactoring, this paper presents a new static data-dependence analysis that identifies necessary critical sections in thread-parallel code that does not yet contain any synchronization between threads. A novel optimization pass then breaks up and shrinks the identified critical sections to maximize parallelism while preserving correctness. Our technique proved to be successful in refactoring sequential assembly-like legacy codes in an industry-sponsored project.

But as refactoring projects are hard to evaluate quantitatively and as the domain specific low-level language is of limited interest, we use a standard benchmark suite for which the optimum, i.e., the minimal set of the necessary atomic block annotations is known. We removed the annotations and let the compiler attempt to rediscover them. For 5 out of 7 benchmarks, our compiler identified the same critical sections as the original programmers did by hand. For the other two benchmarks, the compiler found slightly larger (but also correct) critical sections. In all cases, the versions of the benchmarks that the compiler annotated achieved the original run-time performance.
\end{abstract}

\section{Introduction}

With the increasing use of multicore processors, the refactorization of sequential legacy applications becomes more important. For certain codes the division of the program into multiple threads is relatively easy. This is especially true for low-level control codes in embedded systems where all the concurrent control loops are sequentialized into a (sequential) static schedule. When refactoring such code for use on multicores, the control loops are obvious but it is difficult to identify the necessary critical sections (atomic blocks) to avoid data races, because these have been implicitly implemented in the static schedule or are hidden behind priorities and interrupts. It is likely to miss a critical section, make it too small or too large. If critical sections are implemented with mutexes, it is also too easy to introduce deadlocks.

To solve this problem, we leave the identification of critical sections to the compiler. It analyzes parallel, but unsynchronized code and determines where critical sections are

K. De Bosschere and R. Jhala (Eds.): CC 2013, LNCS 7791, pp. 204-223, 2013.

(C) Springer-Verlag Berlin Heidelberg 2013 
necessary. By means of static data-dependence techniques our algorithm determines the set of variables that concurrent threads share and it also analyzes the correlations between those variables. Of course, every access to a shared variable must be atomic. But correlations between variables also dictate that these variables are to be accessed within an enclosing critical section. Hence, sets of shared variables and correlations among them determine where critical sections need to be placed in the code. We will show that there are two types of correlations which we call explicit and implicit relationships. Explicit relationships can be detected by the compiler by means of dependence analyses. Implicit relationships must be annotated by the programmer. In order to keep critical sections small, a novel optimization pass breaks up the found critical sections into several smaller ones, if possible. In general, these optimizations work by determining which data dependences do not indicate variable correlations and can therefore be ignored by the compiler. Furthermore, we allow a programmer to use annotations that further help to make critical sections smaller.

With this compiler tool at hand, the refactoring workflow for legacy applications is then as follows. A programmer starts to parallelize the code by dividing it into multiple threads, but without adding critical sections. Next, s/he asks the tool to suggest critical sections. In the (rare) presence of implicit relationships, the critical sections may be too small (the compiler might have placed accesses of correlated variables in different critical sections instead of putting them together into one section), but the programmer at least knows all places where races may occur, instead of having to find them on his own. S/He can add annotations to mark implicit relationships in the code. If a suggested critical section seems to be too large, the programmer can think of ways to restructure the code to allow for smaller critical sections or can add annotations to indicate which data dependences can be safely ignored by the compiler analysis. That way, adding synchronization becomes an iterative process that is less error-prone in comparison to having to add all synchronization manually to large legacy code bases.

Another potentially useful application of the techniques presented in this paper would be a checking-tool. Assume a programmer that writes a new parallel program including the critical sections. The checking-tool would ignore these critical sections provided by the programmer, analyze the unsynchronized code, identify critical sections, and present differences between the generated and the programmer-provided critical sections. The programmer can then use this diff to reason about the correctness of his/her solution.

As the industry automation programs we worked with are written in a proprietary domain specific assembly-like language, we present our technique for a broader audience in a $\mathrm{C}$ dialect. We have frontends for both languages that generate the same intermediate representation. The $\mathrm{C}$ frontend is a source-to-source $\mathrm{C}$ compiler that uses POSIX threads to generate parallel code. It accepts $C$ extended with the following extensions for parallel programming. The statement $t=$ spawn $f()$ creates a new thread that starts execution in function $f$ and returns a handle of the thread in $t$. There is also a statement join $t$ that waits until a thread finishes. In order to be able to coordinate the work of multiple threads and to divide it into multiple computational phases, the language also supports barriers. For convenience, the dialect supports a parallel for loop where each iteration spawns a new thread and every thread executes the body of the loop. At the 
end of the loop, there is an implicit join statement that waits for the threads to finish. Note that our compiler does not automatically parallelize the code, it only adds critical sections (atomic blocks) to the output.

In the related work in Sec. 2, we compare our approach to find critical sections to other approaches. We discuss the basic algorithm of our technique in Sec. 3 . Sec. 4 discusses optimizations of the basic algorithm.

When refactoring sequential legacy applications, we cannot judge how well our technique works in practice because we do not know what an optimal refactoring looks like. Hence, in Sec. 5 we evaluate the presented compiler techniques on a standard benchmark suite for which the optimum, i.e., the minimal set of the necessary atomic blocks is known. We use 7 benchmark codes with explicit critical sections. We strip those critical sections from the codes and show that our technique essentially rediscovers the same sections and achieves the same execution times. Annotations are needed in only 2 benchmarks to achieve results similar to the original codes.

The contributions of this paper are (1) a language independent technique that identifies critical sections in the code by analyzing data dependences and a few annotations, and (2) optimizations that further analyze the large number of data dependences in the program in order to detect those dependences that do not indicate correlations between shared variables and therefore do not need to be considered to identify correct critical sections, causing larger sections to be broken up into multiple smaller ones.

\section{Related Work}

The Abstraction-Guided Synthesis of Synchronization (AGS) algorithm of [19] identifies atomic blocks in programs, by means of programmer supplied specifications that describe which program states may not occur. AGS then uses abstract interpretation of the program to find all interleavings that may lead to invalid states. Provably minimal critical sections are added to the program to avoid such interleavings. An incorrect specification results in incorrect atomic blocks. In contrast, our work identifies (potentially non-minimal) critical sections, but it does not need a specification. AGS' critical sections are also only minimal with regard to the abstraction used in the abstract interpretation. AGS also suffers from the state explosion problem that leads to very long compile times. While we tested our work in an industry-sponsored refactorization project and with a full set of standard benchmark codes, AGS is evaluated with small kernels only that neither use pointers nor dynamically allocated memory which we allow.

An algorithm that detects critical sections must know which variables are correlated and therefore need to be accessed within the same critical section. Unfortunately, our approach cannot detect all correlations. We miss so-called implicit relationships, see below, that the programmer needs to express with annotations. MUVI [13] is an orthogonal approach that searches for such variable correlations that are not explicitly specified in the code. While we rely on the programmer to use additional annotations if necessary, MUVI analyzes a program with critical sections for variables that are accessed together. MUVI uses code metrics (like static distance in the code) to classify whether accesses to different variables belong together. As these are heuristics, the analysis can still miss some correlations. If related variables are updated in an inconsistent 
manner or if the accesses appear in two different critical regions, MUVI reports this as an error to the programmer. We could integrate MUVI into our compiler and help the programmer in adding explicit annotations for implicit relationships.

In Data-Centric Synchronization (DCS) [18] a programmer declares synchronization constraints that specify up front which data need to be accessed within the same critical section. The programmer annotates a (Java) class and declares which fields in objects need to be accessed together atomically. DCS then automatically infers critical sections. As object fields cannot be accessed through pointers, DCS can find all places that need synchronization and encloses them in atomic blocks. Colorama [4] is hardware support for DCS. The hardware tracks data accesses and decides when to start a critical section, while our algorithm is a purely static approach that does not need hardware support. In contrast to DCS's explicit declarations, our algorithm derives synchronization constraints from data dependences that are present in the code and only relies on annotations where necessary. While it is easy to derive that a critical section must start before the first access to shared data, DCS does not know when a critical section is allowed to end. DCS assumes that a critical section ends at the end of the function that started the critical section. Our algorithm closes a critical section for all affected variables after the last access to any of them. Both approaches can lead to critical sections that are too short. In our case, a critical section may end too early if there are implicit relationships in the program. In practice however, both the heuristics of DCS and our approach lead to correct sections. Nevertheless, in case of implicit relationships, we could also use the type of annotations used in DCS to help the compiler find smaller critical sections. DCS and Colorama use the same benchmarking method that we do. They remove the synchronization from a given parallel program, let the compiler attempt to rediscover critical sections, and compare the results to the original critical sections in given program. While DCS uses the Java Collections package for its evaluation, Colorama was applied to applications such as Firefox and the MySQL database server. In contrast, we use a standard benchmark suite (STAMP) that is available for C.

Race detection tools examine whether two threads may simultaneously access the same shared memory location without holding a lock, either at runtime like FastTrack [6] or statically like Chord [16]. These tools however only show where races may occur, they do not give any hints on how to prevent them. In contrast, our algorithm suggests how to add synchronization to the code to prevent the potential races.

AtomTracker [15] infers atomic blocks from the code and also detects atomicity violations. While we use static analysis, AtomTracker analyzes the memory traces of several concurrent executions of the program to calculate atomic regions.

Using an escape analysis, the work of Bogda and Hölzle [2] removes unnecessary synchronization from Java objects that are used by only one thread. We use an escape analysis as well to decide beforehand which data is thread-private. We can thus avoid to generate unnecessary atomic blocks.

Our paper focuses on the identification of critical sections and their optimization and not on the mechanics to realize the mutual exclusion. We could either use lock inference or transactional memory to implement the critical sections. Lock inference algorithms analyze programs with critical sections and add explicit lock/unlock statements to the code. Current lock inference approaches scale well for large programs. For example, 
the algorithm of Gudka et al. [7] uses sparse representations of interprocedural dataflow information and various optimizations to speed up dataflow propagations. With transactional memory (TM) [10], critical sections execute atomically inside transactions. A typical implementation records accesses to shared data inside a transaction in a log. At the end of the transaction, the implementation attempts to commit by atomically writing all changes to shared data back to main memory. If another critical section/transaction modified a subset of the shared data that was touched by the committing transaction, the committing transaction aborts and retries to execute the atomic block. For simplicity, we use TM to implement the atomic blocks that our compiler detects to be required for correctness.

\section{Identification of Critical Sections}

The high-level approach of our technique is to detect correlations between shared variables that are used by multiple threads. These correlations determine where critical sections need to be placed in a program. As we will show, there are two classes of correlations, which we call explicit and implicit relationships. Explicit relationships can be detected by dependence analyses, while implicit relationships need to be annotated by the programmer.

To identify critical sections, the compiler must first analyze which statements of the program may execute concurrently to each other. An Andersen-style interprocedural alias analysis (similar to the algorithm of [9]) extended with an escape analysis [17] that is used to identify thread-local data then conservatively approximates the sets of variables that these statements use. The intersection between read and written variables of those sets gives the sets of shared variables. Of course, thread-local variables do not belong to the set of shared variables. In the remainder of this paper, we call a statement of a thread a concurrent instruction if it statically touches shared data. Without synchronization, such statements are prone to race conditions and need to be put into critical sections. Using dependence analyses, our compiler then solves the problem of how concurrent instructions need to be grouped into critical sections, i.e., where critical sections need to start and end.

Sec. 3.1 first discusses correctness issues and limits of our technique to identify critical sections in a program. Sec. 3.2 explains in more detail how the compiler finds concurrent instructions. The algorithm explained in Sec. 3.3 then calculates (large) atomic blocks that guarantee correct synchronization. Sec. 3.4 illustrates the algorithm with an example.

\subsection{Correctness Considerations and Limits}

Let us briefly consider correctness first. Assume a collection of threads that accesses shared variables $a$ to $d$. These variables may or may not affect each other. For example, the value of $b$ may be calculated from the value of $a$, which is a classical flow data dependence [14] that a compiler can detect. An invariant could be that $c$ always has the same value as $d$. Although invariants often are essential for the correctness of the 
program, the compiler in general cannot derive them from the code. Data dependences 1 between shared variables are explicit relationships between variables. A program can also contain invariants/correlations between variables that have no data dependences between. These are correlations implied due to program logic. We call them implicit relationships. Our view of relationships is that once a shared variable $a$ changes its value, all shared variables $b$ related to $a$ must appear to update their values at the same time, i.e., in the same (generated) critical section (instant update policy). If one statement uses several shared variables, we treat the variables as related as well. Hence, all statements that access related variables (explicitly or implicitly) need to execute atomically within the same (generated) critical section.

All the statements $X$ that access related variables are spread over the code. At runtime their critical sections must start before the first of all statements $X$ is executed and must end after the last of them. To statically generate a critical section in the code, the analysis needs to calculate first and last. First dominates 2 all statements $X$ and last post-dominates them. First also dominates last and last post-dominates first. All instructions that lie on a path from first to last also belong to the critical section. First and last therefore correspond to the entries and exits of single-entry-single-exit regions in the program structure tree by Johnson [12].

For programs with solely explicit relationships, our algorithm guarantees correct synchronization. Implicit relations pose limits to a fully automatic approach. Fig. 1 shows two different examples. Within function runl, either one of the two collections $a$ and $b$ holds the element $x$ at all times. To cope with the absence of a data dependence between the collections, the programmer can add an annotation related $(a, b)$ that renders the relationship between $a$ and $b$ explicit. This causes the compiler to

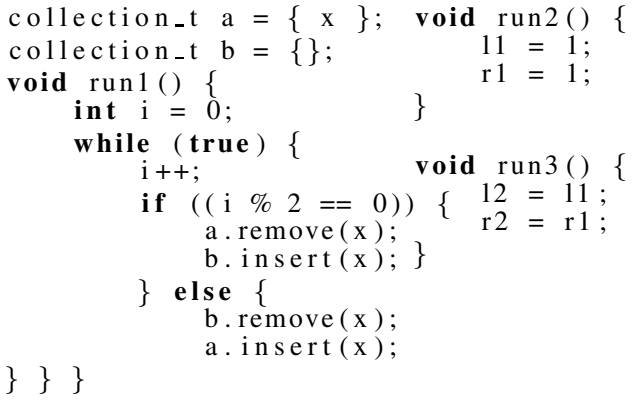

Fig. 1. Implicit relationships put accesses to $a$ and $b$ together into a critical section. In the second example, run 2 and run 3 execute concurrently. In a sequential execution, where run 2 is called before run 3 , the variables $l 1, l 2, r l$, and $r 3$ would have the same value. If run 2 and run 3 executed concurrently, one critical section enclosing the assignments to $l l$ and $r l$, as well as another critical section around the assignments to $l 2$ and $r 2$ are necessary. However, as there are no explicit data dependences between these variables, the analysis cannot establish the necessary synchronization. If it is necessary for the algorithm that $l 1, l 2$, $r 1$, and $r 2$ store the same value, a related annotation is needed to enable the compiler to identify the necessary critical sections. There is also an annotation unrelated $(a, b)$ to explicitly break explicit relationships between variables that are dependent, but that

\footnotetext{
${ }^{1}$ Unless otherwise noted, a dependence is a flow dependence in this paper.

${ }^{2}$ Statement $A$ dominates statement $B$ if $A$ appears on every control flow path from the entry of the function to $B$. $A$ post-dominates $B$ if $A$ appears on every control flow path from $B$ to the exit of the function.
} 
do not need to be in the same critical section. Both types of annotations extend the applicability of our approach in an iterative refactoring tool.

Our approach exploits data dependences and the above annotations to determine critical sections. Note that this includes control dependences, because they are converted into data dependences using techniques from Muchnick [14].

Due to aliases and function pointers the conservative analysis cannot avoid false positive data dependences. These cause larger than necessary (but sill correct) critical sections. While our algorithm already works well on nicely structured programs in legacy languages, modern managed languages offer fewer possibilities to introduce aliases and are therefore even better to analyze, enhancing the applicability of our technique. Furthermore, when our technique is used during the development of new parallel applications, early warnings can indicate too coarse-grained atomic blocks, and help the programmer to improve the code by reducing dependences between variables. As asserts and annotations become more common in new languages, relationship annotations are easy to digest.

Other approaches to infer critical sections such as AGS and DCS always need the programmer to provide explicit specifications in order to work correctly. In contrast, our algorithm works with plain code and just needs annotations in presence of implicit relationships.

\subsection{Concurrency Analysis}

The first step in the identification of critical sections is the analysis that determines which statements may execute concurrently to other statements. Consider the code in Fig. 2 that creates and destroys multiple threads.

To find out which statements may execute concurrently, we use a may-happen-in-parallel analysis [8]. The results of this analysis are given in Fig. 2, The comments show for every statement which other statement may run concurrently to it. For example, after $t$ (function $f$ ) is spawned in main, statement $F$

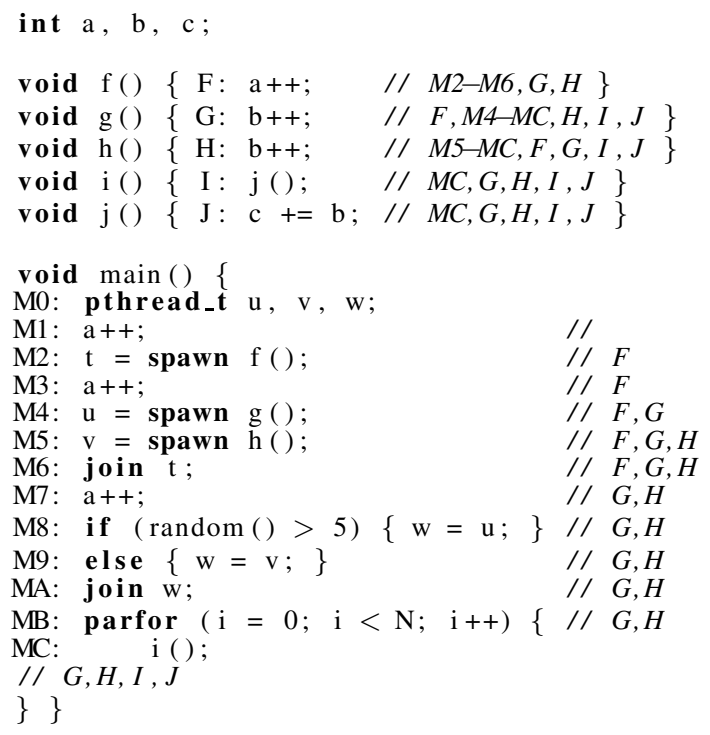

Fig. 2. Concurrency analysis ments $M 2$ to $M 6$ in main up to the join on $t$. On the other hand, $M 2$ to $M 6$ as well as $G$ and $H$ may run concurrently to $F$, because $u$ and $v$ are spawned while $t$ is running. Also note that the parallel for loop spawns multiple instances of $i$. Therefore, $I$ runs concurrently to itself in $i$. As either $u$ or $v$ is still running when 
the parallel for loop is entered, $G$ and $H$ may still execute concurrently to the loop body $(M C)$ as well as concurrently to $I$. As $i$ calls $j$ and multiple instances of $i$ run concurrently to each other, $I$ is concurrent to $J$.

The compiler also analyzes (with a standard alias and escape analysis) which data a thread may touch. For every thread, the compiler examines all statements of the thread and collects all accessed variables in the thread's a read/write set. Given a spawn statement $x=$ spawn $r$, the statements of $x$ are the statements in $r$ plus all the statements in functions that are transitively called by $r$. In the example, the statements of $t, u$, and $v$ are $F, G$, and $H$. The statements that belong to the threads spawned in the parallel for loop are $I$ and $J$. Thread $t$ reads/writes $a$, threads $u$ and $v$ read/write $b$. The threads spawned in the loop read $b$ and write $c$. To determine which data is shared by two threads $x$ and $y$, we intersect the read/write set of $x$ with the write set of $y$ and vice versa. We call the statements that may access shared data and that may execute concurrently to other statements concurrent instructions.

In Sec. 4.4, we will show that an instruction can be falsely identified as a concurrent instruction that is in fact executed by only one thread. In that section, we also illustrate two optimizations that reduce false positives in the identification of concurrent instructions.

\subsection{Basic Algorithm}

We explain the basic algorithm that finds atomic blocks with the pseudo code in Fig. 3 . The algorithm starts by calling get_concurrent_insns to interprocedurally find all concurrent instructions in which two threads may access shared data at the same time. How this is done has been discussed in the previous section.

One aspect worth mentioning is that get_concurrent_inshs also analyzes barriers in the program. Statements in different computational phases separated by a barrier never execute concurrently, since the next phase only starts as soon as all threads have finished their previous phase. Hence, a critical section can never span two computational phases divided by a barrier. An algorithm for the division of code into barrier-separated disjoint phases is given by Jeremiassen et al. [11]. All further steps explained later take those phases into account when they calculate critical sections, but for simplicity we will not mention them any further.

The algorithm then has to bundle all instructions that access related shared variables and all statements that must be executed between those instructions into a single critical section. We call such a bundle of instructions a partition. In the pseudo code, every single concurrent instruction starts a partition of its own that part_intersect and flow will later combine. We describe the optimization remove_superfluous_dependences in Sections 4.1 to 4.3. Part_intersect compares all pairs of partitions to combine those partitions that access a common subset of shared data. Merge calculates the first and last instructions for every partition and also adds all dominated and post-dominated statements in between. Flow compares all pairs of partitions to check whether they have statements with flow dependences/explicit relationships to the data of the partitions and that hence must also be in the merged partition 3

\footnotetext{
${ }^{3}$ There is a flow dependence between two partitions $P$ and $Q$ if there is a statement $S 1$ in $P$ and a statement $S 2$ in $Q$ and there is a path in the dependence graph from $S 1$ to $S 2$.
} 


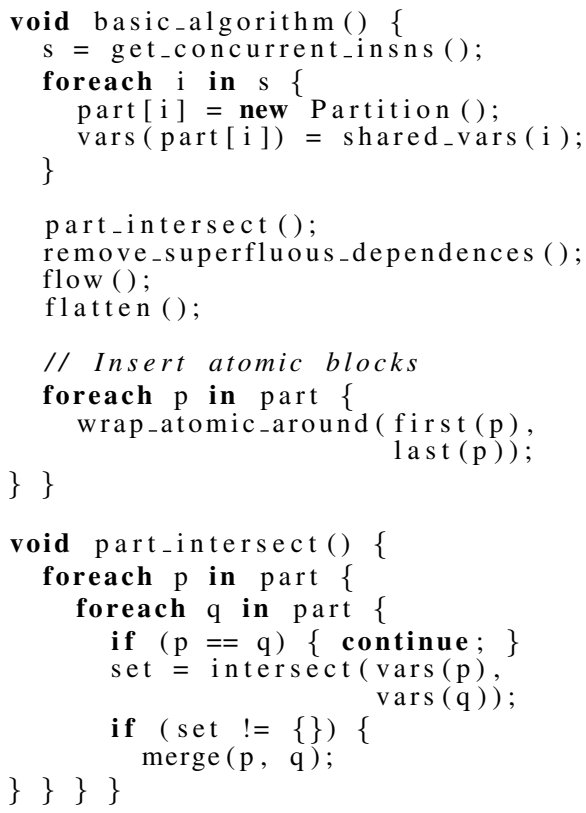

Fig. 3. Basic algorithm

After flow, the resulting partitions are correct critical sections. But they may overlap. Two partitions $p$ and $q$ overlap if on any path from first $(p)$ to $\operatorname{last}(p)$ there is an instruction that belongs to partition $q$ or vice versa. We merge nested or overlapping atomic blocks because most STM implementations do not support them. Flatten therefore performs an intraprocedural depth-first search from the first to the last instruction of a partition. As last post-dominates first, a depth-first search from first will eventually reach last. If on any search path there is an instruction belonging to another partition, the two partitions are merged. After the depth-first search, flatten calls interproc_merge to remove atomic blocks that nest within the call hierarchy. For example, a function $f$ may contain an atomic block that calls $g$, which itself contains an atomic block. The block in $g$ is nested within the block in $f$. An interprocedural analysis walks the call-graph to find call-paths where callees always execute within an atomic block of a caller. Then the callee's atomic blocks can be removed. If $g$ is called both from an atomic context and from a non-atomic context, function cloning generates two versions of $g$. From an atomic context, the version of $g$ without the atomic block is called. Otherwise, the version of $g$ that contains an atomic block is used. Currently, our approach can only handle function pointers if the pointed-to functions provably do not contain atomic blocks. To lift this restriction, the compiler could generate two versions-one version with atomic blocks and one version without - of all functions that have the same signature as the function pointer and use a runtime mechanism to direct calls via function pointers to the proper function in order to avoid nested atomic blocks.

After flattening, each remaining critical section is wrapped into an atomic block. 


\subsection{Sample Execution}

We now apply the algorithm to the example in Fig. 4 Its shared variables are the Nodes that are held in the inputs array, output, next_input, counter, and double_counter. The concurrent instructions are marked with $S 1$ to $S 8$. These are concurrent instructions because the parfor loop created multiple threads that all execute the same function process. Fig. 5 shows the dependence graph of the example's process code. Statements that belong to a partition are marked with $P 1$ to $P 4$.
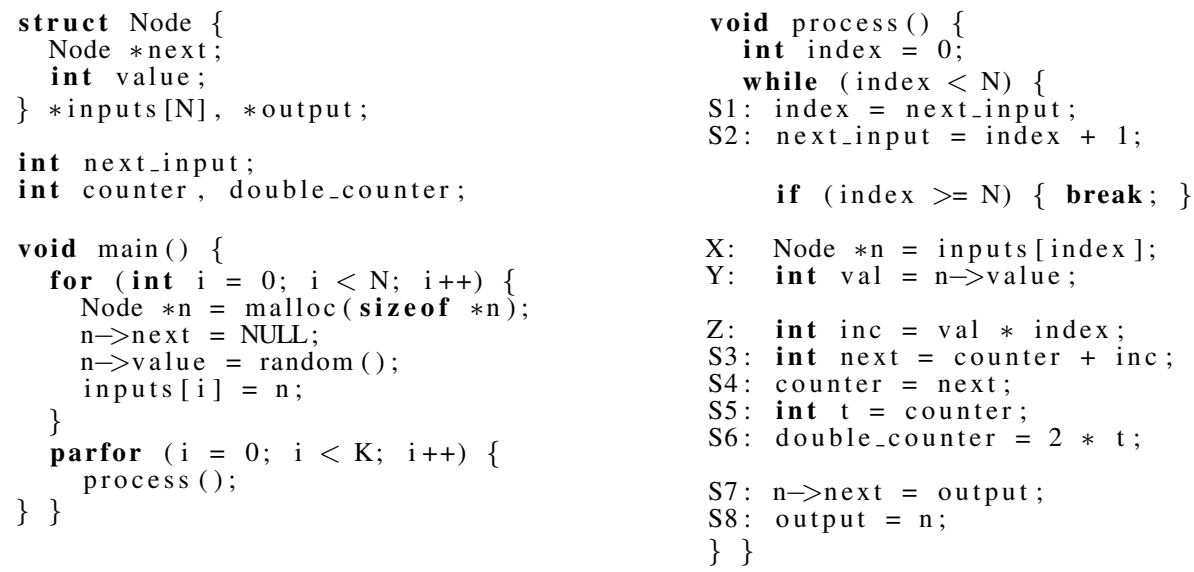

Fig. 4. Example program for atomic block identification

To avoid races, three atomic blocks are needed. First, next_input must be incremented atomically $(S 1, P 1)$. Second, both partitions $P 2$ and $P 3$ must be in a critical section to satisfy the explicit relationship that double_counter $(S 6, P 3)$ is always twice as big as counter ( $S 5, P 2)$. Third, prepending $n$ to the linked list pointed to by output must be atomic. But since all four partitions of Fig. 5 are linked to each other by flow dependences, the basic algorithm combines them into one correct but too large critical section and generates the code in Fig. 64 Sec. 4 presents optimizations that achieve a more finegrained synchronization. No annotations were needed in this example.

\footnotetext{
${ }^{4}$ As there is a chain of dependences between the statements index $=$ next_input (in partition $P 1$ ), inc $=v a l * i n d e x$, and next $=$ counter + inc (in partition $P 2$ ), flow merges $P 1$ and $P 2$. There are additional chains of flow dependences from statements in $P 2$ to instructions in $P 4$, as well as from $P 3$ to $P 4$. Both $P 4$ and $P 3$ join that partition. As there are no overlapping partitions in the running example, flatten shows no effect here.
} 


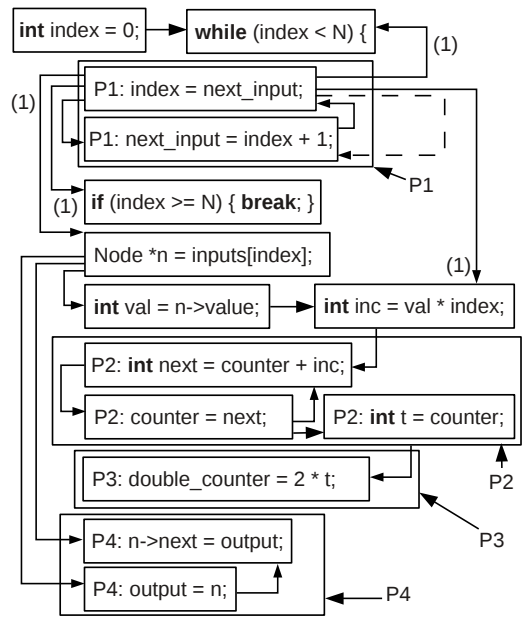

Fig. 5. Example program with flow dependences (full arrows)

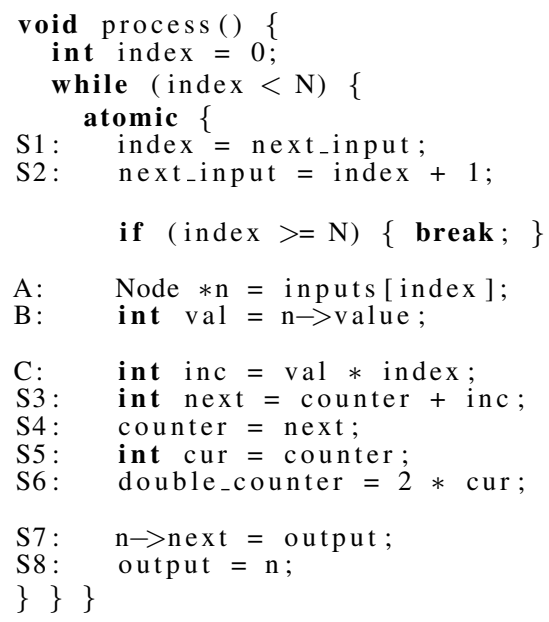

Fig. 6. Synchronized code for Fig. 4

\section{Optimizations}

The basic algorithm calculates a correct synchronization, but it is sometimes too conservative, as flow from Fig. 3 merges any partitions with dependences between them. If we can remove unnecessary dependences between partitions, i.e., dependences that need not be considered to ensure a proper synchronization, large critical sections break up into smaller ones. This allows more parallelism.

For the discussion in this section, always assume that multiple threads execute the code being discussed.

\subsection{Removal of Non-critical Dependences}

Function plain in Fig. 7 writes the shared variable sharedl and copies it to threadlocal variables $x$ and $y$. If executed sequentially, all four variables have the same value after the final assignment to shared2. This invariant can be discovered by analyzing flow dependences. It is necessary for parallel execution and preserves the invariant to put all four flow dependent statements of plain into a single critical section. Assume flow would not have merged the two partitions for the two shared variables. With the resulting two critical sections (around the first two statements and around the final assignment) one thread could modify sharedl while the other assigns the old value of shared 1 that it keeps in $y$ to shared2. This would break the invariant. 
Of course it depends on the application whether it is necessary to satisfy the invariant in a parallel execution. But to conservatively ensure correctness, our technique must make sure that the identified atomic sections satisfy the invariants. If an invariant is not necessary, an annotation could be used. In the example, marking shared 1 and shared 2 as unrelated would lead to different atomic sections.

The code in uncritical is slightly different and demonstrates optimization potential. (Assume that the code is free of implicit relationships as there are no annotations). The code first stores a copy of shared 1 in $x$. Then it increments sharedl. Because the statements work on the same shared variables and since they are flow dependent, the basic algorithm would again create an embracing critical section. But now the discoverable invariant is that the three variables $x, y$, and shared 2 have the same value. There is no explicit relationship between shared 1 and shared 2 anymore. Hence it is sufficient to put the first two assignments into one critical section and to put the assignment to shared2 in a second critical section 5 The assignment to $y$ operates only on thread-local data and thus does not need to be in a critical section at all. Even if a concurrent thread changes shared 1 between those two atomic blocks, $x, y$, and shared 2 will remain unaffected. To generate a correct synchronization, flow therefore can ignore the dependence between the assignment to $x$ and the assignment to $y$.

More formally, the remove_superfluous_dependences optimization (Fig. 3) looks for statements $s$ and $a$ that meet the following criteria: $s$ reads some shared variable, i.e., it belongs to some partition $P$. Statement $a$ postdominates $s$ and is must-antidependent on $s$, i.e., it is guaranteed to overwrite the variable that $s$ has read (shared $1++$ in uncritical). Notice that as $a$ writes the same shared variable, it belongs to $P$ as well. If there are such statements $s$ and $a$, then flow can ignore all outgoing dependence edges of $s$ (the one from $x$ to $y$ in the example).

To see why the optimization is correct, assume that there is a flow dependence between $s$ and another statement $t$. In case part_intersect has already put both $s$ and $t$ into $P$, they belong into the same critical section, no matter what dependences exist between the two statements. In the other case, i.e., if $t$ is outside of $P$, there is room for optimization. If $t$ is in another partition $Q$ or if $s$ and $t$ are part of a dependence

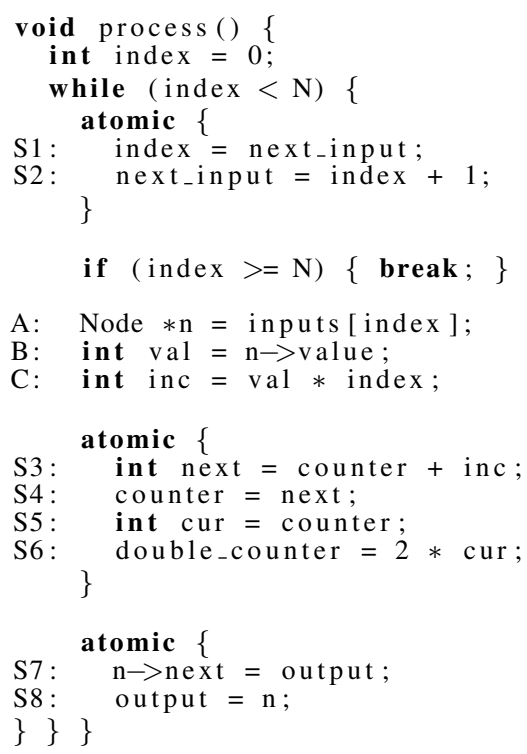

Fig. 8. Optimized result for Fig. 4 chain that ends in another partition $Q$, flow merges $P$ and $Q$ so that $s$ and $t$ are in the same critical section. In the example $t$ is the assignment to $y$ that is linked to the update of shared 2 which is in the second partition. Now the statement $a$ comes into play. As $a$

\footnotetext{
${ }^{5}$ Of course there is an implicit invariant $x==y==$ shared2 $==\operatorname{shared} 1-1$. But this invariant cannot be found by flow dependences (i.e., explicit relationships) only.
} 
belongs to $P$ and post-dominates $s$, and as $t$ is outside of $P$, a always executes between $s$ and $t$ and it always modifies the shared data used by $s$ and $t$. Because $s$ and $t$ are part of a dependence chain from $P$ to $Q$, we cannot construct an invariant from this chain that involves the shared data between $P$ and $Q$, as $a$ always overwrites that data. Thus, the flow dependence edge between $s$ and $t$ does not indicate an explicit relationship and can be removed in flow. If there is no other dependence chain between $P$ and $Q$, these two partitions are not merged and result in separate critical sections.

Remove_superfluous_dependences examines every concurrent statement $s$ in every partition $P$ in some arbitrary order. From $s$ it traverses all outgoing antidependence edges that lead to an antidependent statement $a$. If $a$ post-dominates $s$ and if the results of the alias analysis indicate that $a$ always overwrites the shared data used in $s$, then all outgoing flow dependence edges are removed from $s$

This optimization is applicable to the example of Figs. 4 and 5 After next_input is read and copied to index by $S 1$ (statement $s$ ), next_input is changed by $S 2$ (antidependent statement $a$, dashed arrow). Hence, it is possible to increment next_input in a critical section of its own, as it cannot affect the behavior of flow dependent statements $X$ and $Z$. Note that statement $X$ then still loads $n$ from the proper array element. Flow can ignore all edges marked with (1) in Fig. 5 and only merges partitions $P 2$ and $P 3$ because of data dependences. The resulting code in Fig. 8 has the increment of next_input, the counter updates, and the list prepending in separate critical sections.

\subsection{Removal of Dependences over Read-Only Variables}

We can also safely eliminate dependences over pointer accesses in concurrent instructions that provably only reach read-only data. Assume that a few threads concurrently execute the code with its dependence graph in Fig. 9. The difference to Fig. 4 is that the Nodes are no longer read from an inputs array. Instead, they are removed from a linked list. Part_intersect has identified the partitions $P 1$ and $P 2$. As there is a chain of dependences from $P 1$ to $P 2$, flow would merge those partitions. But it is correct to keep $P 1$ and $P 2$ in two critical sections, because after $n$ is removed

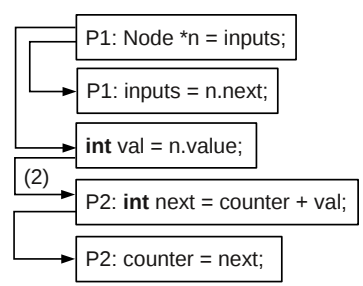

Fig. 9. Dependence over read-only data from inputs, all accesses to it are read-only. No thread could concurrently write to $n$. The only dependence entering $P 2$ from outside comes from a read-only access to $n$. Once the code has retrieved $n$ from inputs, it does not matter how much later the code in $P 2$ is executed, as the memory reachable from $n$ (that $P 2$ depends on) will always contain the same values.

In the example, the edge marked with (2) is removed which causes $P 1$ and $P 2$ to become individual atomic blocks.

Remove_superfluous_dependences examines all flow dependence edges between any two statements $s$ and $s^{\prime}$. If $s^{\prime}$ dereferences a pointer $p$ and if all memory locations transitively reachable from $p$ are read-only (according to the results of the alias analysis), then no concurrent modification between $s$ and $s^{\prime}$ can influence the effects of these two statements. Thus, no matter how long the time span between $s$ and $s^{\prime}$ is, $s^{\prime}$ always appears to execute instantly after $s$. Hence, if $s$ and $s^{\prime}$ are linked in a dependence chain 
that starts in a partition $P$ and that ends in another partition $Q$, an update of shared variables in $P$ always appears to happen instantly with the corresponding update in $Q$, even if $P$ and $Q$ belong to different critical sections. This allows flow to safely remove the dependence between $s$ and $s^{\prime}$. If there is no other dependence chain between $P$ and $Q$, these two partitions can execute in separate critical sections.

In the example, all threads executed the same function and the fields of $n$ were never written. Recall from Sec. 3.2 that the compiler analyzes which instructions may execute concurrently. In the above analysis, if the compiler finds an instruction that accesses $p$, it checks which set $T$ of threads may execute concurrently to this instruction. If all threads in $T$ only read all memory locations transitively reachable from $p$, then the optimization above is correct. That means, threads that write to memory locations reachable from $p$ are still possible. As long as those threads are not in $T$, the optimization is applicable.

\subsection{Removal of Dependences between Builtin Data Structures}

In Fig. 10 a pop routine of a handwritten stack is called. There is a chain of dependences from the internal representation of the stack (an array of items) to the access of the returned item after the pop. This forces all the statements into a single critical section. In contrast, with opaque thread-safe library data structures with known semantics as

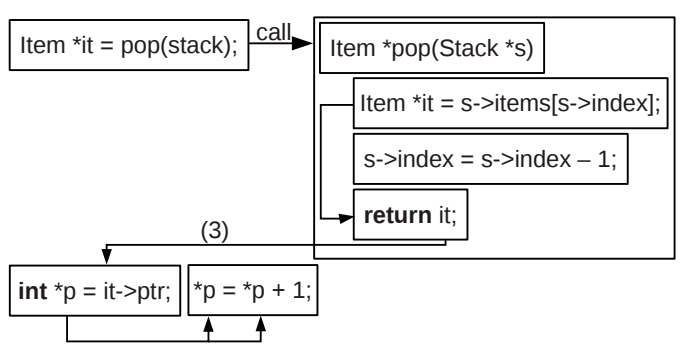

Fig. 10. Dependences over a builtin data structure they are common in modern languages (Java, Python, etc.), there is no such dependence chain, resulting in two smaller critical sections one for pop and one for the access to the popped item.

Note that compiler analysis is sped up as well because the library codes do not need to be analyzed. For this optimization to be correct, there may be no implicit relationships between a black box builtin container data structure and and other shared data. Otherwise related annotations are needed. Remove-_super-fluous_dependences finds all calls of functions that are known to be thread-safe and removes outgoing dependences from those calls. In the example, the edge marked with (3) is removed.

\subsection{Detection of Concurrent Instructions That Execute in a Single Thread}

All threads that execute the code in Fig. 11 share $V$ and $A$. Although the statements $S 1$ to $S 3$ appear to be concurrent instructions, $S 1$ is not because only the thread with ID 0 executes it. $S 3$ is neither a concurrent instruction as all threads access different elements of $A$ (threadId() is unique for each of them).

Hence, to eliminate concurrent instructions that run in only one thread, we first check if there is a computational phase that matches the construct

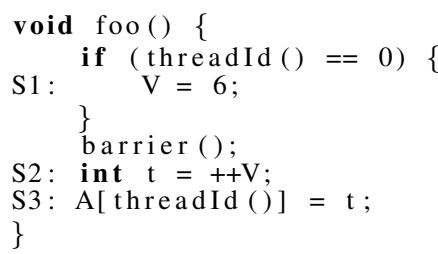

Fig. 11. Concurrent instructions executing in one thread 
if $($ threadId ()$==x)\{\ldots\}$. Then only one thread executes the body which is thus free of concurrent instructions. Second, two statements accessing the same array are considered concurrent instructions unless their subscript expressions have the form $a *$ threadId ()$+b$, with constant values for $a \neq 0$ and $b$. Is this case we know that every thread accesses a different array element. Dependence analysis as used in loop parallelization [1] can further improve subscript analysis.

\section{Evaluation}

Methodology. Although we have developed our techniques for a refactoring tool, we discuss an evaluation that uses a parallel benchmark suite. The evaluation of our approach is designed to show that (a) the identified critical sections are correct and small, and (b) the execution times of the code generated by our compiler match the execution times of code written by an expert programmer. We let our compiler analyze programs from STAMP [3], a benchmark suite for testing transactional memory implementations. The code contains atomic sections that permit as much concurrency as possible. For our evaluation, we remove the atomic start and atomic end statements and use the resulting unsynchronized thread-parallel codes as input.

We use this method of evaluation because it is hard to evaluate how good a refactoring tool is. For a given sequential legacy application it is simply unknown what the best parallel refactorization is. Thus there is no way to compare the results of a tool to some unknown optimal result. We could therefore make no sound statements about the quality of our approach.

Therefore, we compare the number and sizes of the atomic blocks in STAMP with the atomic blocks generated by our compiler. If they match, our algorithm works. In case of variations, the runtime effects are interesting. Hence, we also compare execution times with SwissTM [5] as target platform for the measurements. We also use STM instead of lock inference because STAMP is an STM benchmark that does not have lock/unlock statements in the code either. Because the critical sections in STAMP are (almost) minimal, if an automated approach can identify identical critical sections and generate code that is equally fast, the automatic approach is good.

This general evaluation methodology to use existing programs, remove all the synchronization that is present in the codes, and then let the tool try to rediscover them is also used in the evaluation of DCS [18] and Colorama [4].

We ran all benchmarks on a $2.66 \mathrm{GHz}, 8$ core Xeon (X5550) with $8 \mathrm{MB}$ cache and 24 GB main memory, with Linux 2.6, using one, two, four, and eight cores. We excluded the $S s c a 2$ benchmark as even the original STAMP version already always crashed in our setup.

Setup and Measurements. Table 1 holds the lines of code, the times $t_{A}$ for the alias analysis, $t_{E}$ for escape analysis, $t_{D}$ for the initial construction of the dependence graph, $t_{I}$ for the identification of atomic blocks, and $t_{T}$ for the total compile time. As we ported STAMP to our C dialect first, we report the number of lines of the ported versions. Column orig contains the original number of atomic blocks in STAMP, columns gen and gen $n_{\text {opt }}$ give the number of blocks identified by our basic algorithm and with optimizations enabled. Column opts lists the applicable optimizations (sub-section numbers). 
Table 1. Lines of code, compile times, and atomic block details

\begin{tabular}{l|r|r|r|r|r|r|r|r|r|r} 
Benchmark & Lines & $t_{A}$ & $t_{E}$ & $t_{D}$ & $t_{I}$ & $t_{T}$ & orig & gen & gen & opt \\
\hline Vacation & 504 & $0.09 \mathrm{~s}$ & $0.02 \mathrm{~s}$ & $0.15 \mathrm{~s}$ & $0.01 \mathrm{~s}$ & $0.27 \mathrm{~s}$ & 3 & 3 & 3 & none \\
Intruder & 3266 & $0.97 \mathrm{~s}$ & $1.66 \mathrm{~s}$ & $0.55 \mathrm{~s}$ & $0.04 \mathrm{~s}$ & $3.28 \mathrm{~s}$ & 3 & 2 & 3 & $4.3,4.4$ \\
\hline Bayes & 5856 & $1.23 \mathrm{~s}$ & $0.33 \mathrm{~s}$ & $0.77 \mathrm{~s}$ & $0.14 \mathrm{~s}$ & $2.47 \mathrm{~s}$ & $2+13=15$ & $2+1=3$ & $2+3+1=6$ & $4.3,4.4$ \\
& & & & & & & & & & \\
$2+13+1=16$ & + annot. \\
Yada & 6292 & $0.49 \mathrm{~s}$ & $0.97 \mathrm{~s}$ & $1.09 \mathrm{~s}$ & $0.14 \mathrm{~s}$ & $2.69 \mathrm{~s}$ & 6 & 3 & 5 & 4.3 \\
& & & & & & & & & 7 \\
\hline Genome & 1861 & $0.19 \mathrm{~s}$ & $0.04 \mathrm{~s}$ & $0.33 \mathrm{~s}$ & $0.18 \mathrm{~s}$ & $0.74 \mathrm{~s}$ & 5 & 7 & 5 & 4.4 \\
Labyrinth & 2880 & $0.21 \mathrm{~s}$ & $0.04 \mathrm{~s}$ & $0.46 \mathrm{~s}$ & $0.01 \mathrm{~s}$ & $0.72 \mathrm{~s}$ & 3 & 3 & 4 & 4.2 \\
Kmeans & 742 & $0.08 \mathrm{~s}$ & $4.03 \mathrm{~ms}$ & $0.16 \mathrm{~s}$ & $3.68 \mathrm{~ms}$ & $0.25 \mathrm{~s}$ & 3 & 2 & 4 & $4.1,4.4$
\end{tabular}

To enable optimization 4.3 in STAMP, we treat its container structures such as lists, heaps, etc. as builtin.

Fig. 12 shows the improvements of the execution times of the optimized over the unoptimized code generated by our compiler and compares the runtimes of the optimized code to the original STAMP versions.

General Results. First, and most importantly, our algorithm generates correctly synchronized programs. Second, the number of atomic blocks found by our algorithm with optimizations enabled ( gen $_{\text {opt }}$ ) is in good accordance with the number of atomic blocks present in the original benchmarks (orig). We will discuss differences below. Third, even if the optimizations do not lead to the same blocks, the execution times of our code are similar to the original benchmarks. Fourth, the optimizations are essential because the execution times of the programs generated without them are significantly slower. These results show that our approach is helpful for programmers.

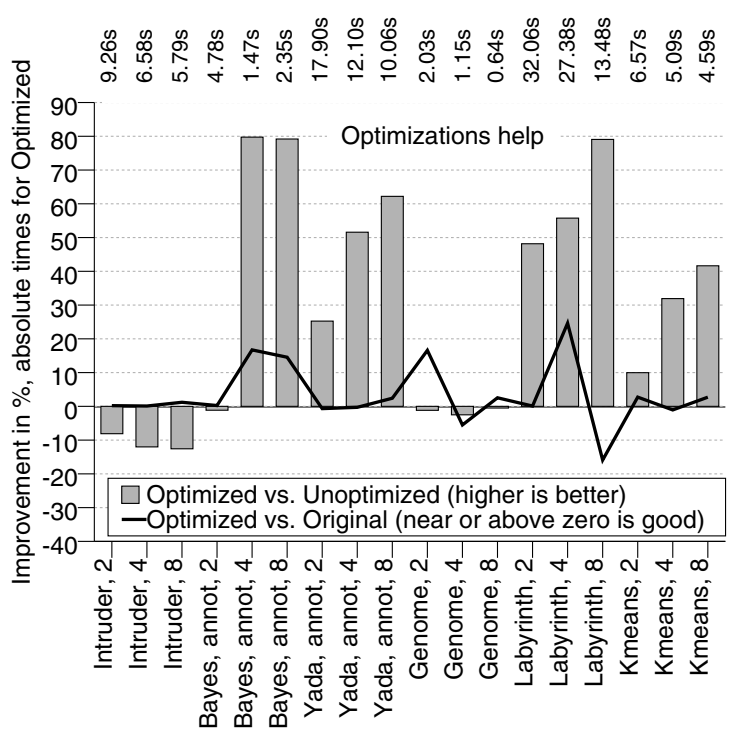

Benchmark, number of cores

Fig. 12. Execution times

Compile Times. With at most 3.3 seconds they are good and an improvement over the related AGS algorithm (see Sec.2), which reported compile times to be less than 10 minutes for algorithmic kernels instead of full benchmarks. The time for the identification 
of atomic blocks generally is between $1.2 \%$ and $24.3 \%$ of the overall compile time. The alias, escape and dependence analyses are slowest but improvable, as we use an unoptimized $O\left(n^{3}\right)$ Andersen style alias analysis, a reaching definitions analysis to calculate dependences, and an unoptimized implementation of an escape analysis 6 We did not invest in more advanced state-of-the art analyses as the basic ones were sufficient and let us focus on the identification of critical sections.

We now examine the results of our algorithm and the execution times for the individual benchmarks. We skip single core results as atomic blocks do not matter in absence of parallelism.

Perfect Matches. Our compiler finds exactly the original atomic blocks for Intruder and Vacation. For Vacation no optimizations are needed. So we skip the runtime numbers as there is no effect to be seen. For Intruder, the version with optimization has the same speed as the original code. Surprisingly, without optimizations ( 2 atomic blocks), the code with 3 atomic blocks is between $7.5 \%$ and $11.2 \%$ faster. Fewer and larger atomic blocks are beneficial for STM performance if transaction setup can be saved for tiny and adjacent atomic blocks. This result shows potential for STM optimizations and improvements of the atomic block annotations in STAMP.

Good Matches; Annotations Needed. For Bayes and Yada we discover all atomic blocks as they are in STAMP, which then leads to the same execution times. But we need some annotations. Bayes turns out to be the hardest benchmark. It contains two computational phases. Let us first discuss the results without annotations. Phase (1) creates a list of tasks that are then used by phase (2). Phase (1) has two atomic blocks which our compiler detects. The first block updates a global variable, the second block adds a task to the list. The difference to the original STAMP code is that our compiler puts the task creation into the atomic block, as it falsely assumes that the task is shared at that point. But this does not cause any slowdown. Phase (2) contains 13 atomic blocks in STAMP. Our compiler generates 3 larger blocks (plus one unnecessary block) because it has to conservatively respect some dependences. With added pseudo-barrier annotations, the 3 blocks are split into 13 separate critical sections. A pseudo-barrier is a no-op at runtime but like for an ordinary barrier, the analysis exploits that atomic sections cannot span barriers. The superfluous block is small. It holds an access to the task data structure that is thread-private at that point. This is harmless at runtime as the logging overhead of the STM is negligible and as the task structure at that point is thread-private. The optimizations are important, as they improve runtime by $79.8 \%$ to $79.2 \%$ over the unoptimized code. But the additional transaction setup overhead of more atomic blocks can only be amortized at higher thread counts.

Annotations are important for Yada as well. The original Yada code has six atomic blocks. The shared data structures are a mesh, elements of the mesh, a heap, and two global variables. Every thread executes a loop that contains the atomic blocks. Block (1) removes an element from the heap. Block (2) checks if the element is invalid. If it is, the code starts another loop iteration. Block (3) refines the mesh. Block (4) marks the

\footnotetext{
${ }^{6}$ Our escape analysis interprocedurally propagates the escape information until a fix-point is reached. Propagation starts at the main function of the program. If there is a call to a function $f$ in the program, we (repeatedly) visit $f$ to propagate the information, which is costly.
} 
element removed from the heap as unreferenced. Block (5) adds elements recognized as bad during the execution back to the heap. After the loop, block (6) updates two global variables. With optimizations enabled, we exactly rediscover blocks (1) and (2). As blocks (3)-(5) share data dependences and touch the same data, part_intersect and flow merge them into one large block. Again, pseudo-barrier annotations help to split this block into the original three parts. The generated code runs at the same speed as the original STAMP code. As block (6) updates two different global variables, our compiler inserts two correct atomic blocks here. The variables are independent and they are only used by the main program once the threads exit. Again, the optimizations are important as the code is between $25.3 \%$ and $62.2 \%$ faster than the unoptimized version.

Close Matches with Irrelevant Variations. For the remaining three benchmarks, our compiler generates essentially the same atomic blocks as the STAMP codes if optimizations are enabled. This results in identical execution times.

For Genome our technique generates similar atomic blocks as the original version. There are three minor differences in the generated blocks. First, the benchmark performs a number of atomic insertions to a hash table in one loop. The original version puts the atomic block around the loop, probably because transaction setups are costly. Our compiler puts the atomic block inside the loop, which is functionally equivalent. Second, the original code has two atomic blocks that call a table insertion routine. Our generated code has the atomic blocks in the insertion routine, which is essentially the same. Third, the third block in our version is a a superfluous block similar to Bayes where the compiler conservatively assumes that a statement touches shared data. At the statement, the variable has become thread-private however, which the analysis does not recognize. As this atomic block is small, its overhead at runtime is negligible for the same reasons as for the extra block in Bayes. The execution times are essentially the same. If the atomic block runs in the loop, the transactional logs per iteration are smaller and lookups to it are faster. These savings outweigh the additional transaction creation setup. Again, on two cores there is not enough parallelism to smooth over different transaction setup costs, so that our code runs faster than the original STAMP code. Without optimizations, there are two additional blocks executed by only one thread. As they are small and transaction conflicts are impossible, the optimized and unoptimized versions have the same execution times.

Our technique generates more and smaller atomic blocks for Labyrinth and Kmeans. We re-discover all original atomic blocks in Labyrinth plus one additional block. This block is added to an insert method of a linked list that also increments the size field of the list. As there are no dependences (i.e., explicit relationships) between the pointer update and the size increment, our compiler puts these two parts of the code in separate atomic blocks. As Labyrinth does not use the list's size field, it runs correctly with this change. Otherwise, the programmer would have to mark the list entries and the size field as related. The small overhead of the additional block shows little runtime effects except for occasional jitter. The optimizations improve runtimes by $48.2 \%$ to $79.1 \%$.

For Kmeans we generate 4 instead of the original 3 atomic blocks. Fig. 13 shows where the difference comes from. Nevertheless, the execution times are identical. The threads share the arrays len and $C$. Our finer synchronization is correct as len and $C$ are independent, and the loop iterations are independent as well. STAMP uses one atomic 


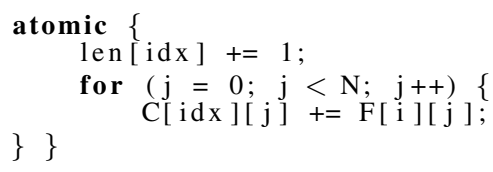

(a) STAMP

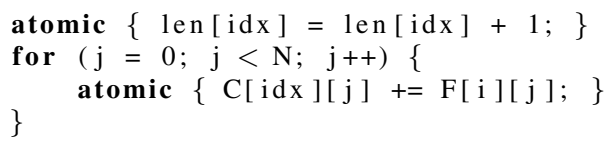

(b) Our output

Fig. 13. Atomic blocks in STAMP and found by our technique

block outside of the loop probably because transaction setup is assumed to be costly. Again, optimizations proved to be effective because they make the code run between $10.0 \%$ and $41.6 \%$ faster than the unoptimized version.

\section{Conclusions and Future Work}

To aid programmers in adding critical sections to parallel code, (or even take this burden off of the programmer), we presented an algorithm that detects critical sections in parallelized, but unsynchronized code. We used our technique in a refactoring tool for embedded industry automation applications written in a low-level language.

Whereas previous approaches to detect critical sections always needed explicit specifications in order to be able to infer critical sections, our technical contribution is an algorithm that starts from the data dependences in the plain code (and only needs some annotations) and discovers the necessary critical sections. Several important optimizations have been presented that help to keep the atomic blocks small. These optimizations detect edges in the dependence graphs that do not need to be considered by the algorithm to detect correct critical sections.

We used the STAMP benchmarks to show that our approach often detects the same set of atomic blocks that an expert programmer would add to the code. In most cases, no annotations were needed in order to generate a correct synchronization. Implicit relationships between variables seldom are a problem for our approach in practice. The execution times for the codes generated by our compiler almost always match the execution times of the manually synchronized versions.

Future work based on the presented technique can address a tool that helps the programmer to check manual synchronization for correctness.

\section{References}

1. Banerjee, U.: Dependence Analysis. Kluwer Academic Publishers, Norwell (1997)

2. Bogda, J., Hölzle, U.: Removing unnecessary synchronization in java. In: OOPSLA 1999: Proc. ACM SIGPLAN Conf. on Object-Priented Programming, Systems, Languages, and Applications. Denver, Co., ACM (November 1999)

3. Cao Minh, C., Chung, J., Kozyrakis, C., Olukotun, K.: STAMP: Stanford transactional applications for multi-processing. In: IISWC 2008: Proc. IEEE Intl. Symp. Workload Characterization, Seattle, WA, pp. 35-46 (September 2008) 
4. Ceze, L., Montesinos, P., von Praun, C., Torrellas, J.: Colorama: Architectural support for data-centric synchronization. In: HPCA 2007: Proc. IEEE Intl. Symp. High Performance Computer Architecture, Phoenix, AZ, pp. 133-144 (February 2007)

5. Dragojević, A., Guerraoui, R., Kapalka, M.: Stretching transactional memory. In: PLDI 2009: Proc. ACM SIGPLAN Conf. Programming Lang. Design and Impl., Dublin, Ireland, pp. 155-165 (June 2009)

6. Flanagan, C., Freund, S.N.: Fasttrack: efficient and precise dynamic race detection. In: PLDI 2009: Proc. ACM SIGPLAN Conf. on Programming Language Design and Implementation, Dublin, Ireland, pp. 121-133 (June 2009)

7. Gudka, K., Harris, T., Eisenbach, S.: Lock Inference in the Presence of Large Libraries. In: Noble, J. (ed.) ECOOP 2012. LNCS, vol. 7313, pp. 308-332. Springer, Heidelberg (2012)

8. Halpert, R.L., Pickett Christopher, J.F., Clark, V.: Component-based lock allocation. In: PACT 2007: Proc. 16th Intl. Conf. on Parallel Architecture and Compilation Techniques, Brasov, Romania, pp. 353-364 (September 2007)

9. Heintze, N., Tardieu, O.: Ultra-fast aliasing analysis using CLA: a million lines of C code in a second. In: PLDI 2001: Proc. ACM SIGPLAN Conf. on Programming Language Design and Implementation, Snowbird, UT, pp. 254-263 (June 2001)

10. Herlihy, M., Moss, J.E.B.: Transactional memory: architectural support for lock-free data structures. ACM SIGARCH Comput. Archit. News 21(2), 289-300 (1993)

11. Jeremiassen, T., Eggers, S.J.: Static analysis of barrier synchronization in explicitly parallel programs. In: PACT 1994: Proc. IFIP WG 10.3 Working Conf. on Parallel Architectures and Compilation Techniques, pp. 171-180 (August 1994)

12. Johnson, R., Pearson, D., Pingali, K.: The program structure tree: Computing control regions in linear time. In: PLDI 1994: Proc. ACM SIGPLAN Conf. on Programming Language Design and Implementation, Orlando, FL, pp. 171-185 (June 1994)

13. Lu, S., Park, S., Hu, C., Ma, X., Jiang, W., Li, Z., Popa, R.A., Zhou, Y.: MUVI: automatically inferring multi-variable access correlations and detecting related semantic and concurrency bugs. In: SOSP 2007: Proc. ACM SIGOPS Symp. Operating Systems Principles, Stevenson, WA, pp. 103-116 (October 2007)

14. Muchnick, S.: Advanced Compiler Design And Implementation. Morgan Kaufmann Publishers Inc., San Francisco (1997)

15. Muzahid, A., Otsuki, N., Torrellas, J.: AtomTracker: A comprehensive approach to atomic region inference and violation detection. In: MICRO 43: Proc. 43rd Annual IEEE/ACM Intl. Symp. Microarchitecture, Atlanta, GA, pp. 287-297 (December 2010)

16. Naik, M., Aiken, A., Whaley, J.: Effective static race detection for java. In: PLDI 2006: Proc. ACM SIGPLAN Conf. on Programming Language Design and Implementation, Ottawa, ON, Canada, pp. 308-319 (June 2006)

17. Salcianu, A., Rinard, M.: Pointer and escape analysis for multithreaded programs. In: PPoPP 2001: Proc. ACM SIGPLAN Symp. Principles and Practices of Parallel Programming, Snowbird, UT, pp. 12-23 (June 2001)

18. Vaziri, M., Tip, F., Dolby, J.: Associating synchronization constraints with data in an objectoriented language. In: POPL 2006: Proc. ACM SIGPLAN-SIGACT Symp. Principles of Programming Languages, Charleston, SC, pp. 334-345 (January 2006)

19. Vechev, M., Yahav, E., Yorsh, G.: Abstraction-guided synthesis of synchronization. In: POPL 2010: Proc. ACM SIGPLAN-SIGACT Symp. Principles of Programming Languages, Madrid, Spain, pp. 327-338 (January 2010) 